MITSUBISHI ELECTRIC RESEARCH LABORATORIES

http://www.merl.com

\title{
Opportunities and Potential of Model Predictive Control for Low-Thrust Spacecraft Station-Keeping and Momentum-Management
}

\author{
Weiss, A.; Di Cairano, S. \\ TR2015-083 July 2015
}

\begin{abstract}
While electric propulsion generates fuel-efficient thrust for spacecraft control, it can only produce low levels of thrust, necessitating continuous actuation to impart an equivalent impulse to that of chemical thrusters. Thus, many of the standard open-loop propulsion scheduling techniques, developed for impulsive thrust, do not transfer to low-thrust architectures. Continuous actuation, together with tighter anticipated requirements for spacecraft station keeping, e.g., in geostationary Earth orbit (GEO), provides great opportunity for the application of feedback control. We demonstrate that model predictive control (MPC) can provide significant advantages as a control strategy for station keeping of low-thrust spacecraft, provided that its features, such as the capability to enforce output constraints, and the use of a prediction model for the plant and disturbances, are fully exploited. We develop a basic MPC design for station keeping in GEO, and compare its performance with an advanced MPC design that i ludes output constraints and disturbance prediction. We show that the basic MPC achieves precise regulation, albeit with unsustainable fuel consumption, whereas the advanced MPC satisfies the target mission requirements with fuel consumption in line with that of carefully designed open-loop strategies.
\end{abstract}

2015 European Control Conference (ECC) 2015

This work may not be copied or reproduced in whole or in part for any commercial purpose. Permission to copy in whole or in part without payment of fee is granted for nonprofit educational and research purposes provided that all such whole or partial copies include the following: a notice that such copying is by permission of Mitsubishi Electric Research Laboratories, Inc.; an acknowledgment of the authors and individual contributions to the work; and all applicable portions of the copyright notice. Copying, reproduction, or republishing for any other purpose shall require a license with payment of fee to Mitsubishi Electric Research Laboratories, Inc. All rights reserved. 



\title{
Opportunities and Potential of Model Predictive Control for Low-Thrust Spacecraft Station-Keeping and Momentum-Management
}

\author{
Avishai Weiss and Stefano Di Cairano
}

\begin{abstract}
While electric propulsion generates fuel-efficient thrust for spacecraft control, it can only produce low levels of thrust, necessitating continuous actuation to impart an equivalent impulse to that of chemical thrusters. Thus, many of the standard open-loop propulsion scheduling techniques, developed for impulsive thrust, do not transfer to low-thrust architectures. Continuous actuation, together with tighter anticipated requirements for spacecraft station keeping, e.g., in geostationary Earth orbit (GEO), provides great opportunity for the application of feedback control. We demonstrate that model predictive control (MPC) can provide significant advantages as a control strategy for station keeping of low-thrust spacecraft, provided that its features, such as the capability to enforce output constraints, and the use of a prediction model for the plant and disturbances, are fully exploited. We develop a basic MPC design for station keeping in GEO, and compare its performance with an advanced MPC design that includes output constraints and disturbance prediction. We show that the basic MPC achieves precise regulation, albeit with unsustainable fuel consumption, whereas the advanced MPC satisfies the target mission requirements with fuel consumption in line with that of carefully designed open-loop strategies.
\end{abstract}

\section{INTRODUCTION}

Spacecraft equipped with electric thrusters generate force more efficiently with respect to propellant mass than those with conventional chemical thrusters, enabling increased spacecraft longevity, larger payloads, and/or cheaper orbital insertion. Conversely, electric propulsion produces relatively low thrust, necessitating continuous thruster firing, and thus requiring different control strategies. While continuous actuation complicates maneuvers that require large impulse, it is suitable for maneuvers that require small forces and torques, and thus opens the possibility to equip a spacecraft with a single set of multipurpose thrusters for orbital station keeping, attitude control, and momentum management.

A further area of research in spacecraft control is the use of on-board feedback rather than the standard open-loop approach that is scheduled from the ground (see, e.g., [1]). For small spacecraft, ground-based control action can be the dominant element in terms of cost and risk [2]. On-board feedback control increases spacecraft autonomy and may also achieve finer regulation of spacecraft orbit and attitude, which is beneficial in light of tighter anticipated spacecraft operational requirements, e.g., the state-space region where the spacecraft must be maintained for correct operation.

Interest in model predictive control for spacecraft applications has developed due to MPC's capability of controlling multi-input, multi-output systems subject to constraints,

A. Weiss and S. Di Cairano are with the Mitsubishi Electric Research Laboratories, Cambridge, MA 02139, USA, weiss@merl.com, dicairano@ieee.org. while optimizing a user defined cost function based on a predictive model for the system dynamics. The ability to explicitly handle multivariable systems, constraints, and performance objectives has rendered MPC very attractive in various fields such as automotive, factory automation, and spacecraft control [3], [4], where it has been proposed for orbital rendezvous [5]-[7], attitude control [8], formation flight [9], and interplanetary transfer [10]. Even though MPC requires higher computational load than conventional closedloop controllers (e.g., PID), linear-quadratic MPC results in quadratic programs that can be solved quickly and efficiently in resource constrained hardware by compact and easyto-verify algorithms, see, e.g., [11] and references therein. However, to provide superior performance, the distinguishing characteristics of MPC (i.e., prediction, constraints, performance optimization) must be properly exploited in the context of the application, otherwise the closed-loop performance may not surpass (and may even be significantly inferior to) that of more conventional techniques.

We demonstrate the potential and opportunity of MPC for low-thrust spacecraft by using as a case study the simultaneous station keeping and momentum management of a nadir-pointing spacecraft in geostationary Earth orbit (GEO). Station keeping (SK) involves the compensation of external perturbations so that the spacecraft remains in an appropriate station keeping window, that is, a rectangular box of longitude and latitude above the Earth [2]. MPC based on an orbital element prediction model was investigated for SK in [13]. Momentum management (MM) is the operation in which the on-board momentum exchange devices, commonly used for attitude control, are decelerated to prevent angular momentum saturation and the subsequent loss of spacecraft attitude regulation capability [12]. The torque due to deceleration must be compensated by the on-board thrusters in order to maintain the nadir-pointing attitude.

The paper is organized as follows. In Section II we model the orbital dynamics and the attitude dynamics of lowthrust spacecraft and describe the simultaneous SK and MM (SK-MM) requirements. We exploit the dynamics model to derive a basic MPC policy in Section III, fairly similar to an LQR design. The basic MPC satisfies some, but not all, of the requirements of the case study because it does not entirely exploit the capabilities of MPC. When this is done in the more advanced design presented in Section IV, all the requirements are achieved, resulting in closed-loop performance that has similar fuel consumption to that of well-studied optimal open-loop control techniques, yet enforcing a 5-10 times smaller SK window. We summarize 
our conclusions in Section V.

\section{SpaceCraft Station KeEPing AND MOMENTUM MANAGEMENT}

We consider a spacecraft equipped with six dual-axis electric thrusters and three axisymmetric reaction wheels attached to a rigid bus in an orthogonal and mass balanced configuration. We assume that a bus-fixed frame is defined for the spacecraft, and that an inertial frame is specified for determining the attitude of the spacecraft. The spacecraft equations of motion are given by

$$
\begin{aligned}
\ddot{r} & =-\mu \frac{r}{|r|^{3}}+\frac{1}{m} F+a_{\mathrm{p}}, \\
J \dot{\omega} & =\left(J \omega+J_{\alpha} \nu\right) \times \omega-J_{\alpha} \eta+\tau, \\
\dot{\nu} & =\eta, \\
\dot{R} & =R \omega^{\times},
\end{aligned}
$$

where $r \in \mathbb{R}^{3}$ is the position vector of the spacecraft with respect to the center of the Earth, $F \in \mathbb{R}^{3}$ is the vector of external forces applied to by the thrusters, $a_{\mathrm{p}} \in \mathbb{R}^{3}$ is the vector of perturbation accelerations, $m$ is the mass of the spacecraft, $\mu$ is Earth's gravitational constant, $J \in \mathbb{R}^{3 \times 3}$ is the moment of inertia of the spacecraft bus and reaction wheel array, $J_{\alpha} \in \mathbb{R}^{3 \times 3}$ is the moment of inertia of the reaction wheel array, $\omega \in \mathbb{R}^{3}$ is the angular velocity of the bus frame with respect to the inertial frame, $\nu \in \mathbb{R}^{3}$ is the angular velocity of the reaction wheel array, $\tau \in \mathbb{R}^{3}$ is the torque applied by the thrusters, $\omega^{\times}$is the cross-product matrix of $\omega$, and $R \in \mathbb{R}^{3 \times 3}$ is the rotation dyadic that transforms the inertial frame into the bus frame resolved in the bus frame. See [14] for the derivation of (1).

For a spacecraft in GEO, the main perturbation accelerations are due to solar and lunar gravitational attraction, solar radiation pressure, and the anisotropic geopotential, that is, Earth's non-spherical gravitational field. Analytic expressions for these perturbation forces per unit mass, i.e., the disturbance accelerations, are given, respectively, by

$$
\begin{aligned}
\vec{a}_{\text {sun }} & =\mu_{\text {sun }}\left(\frac{\vec{r}_{\text {sun } / \mathrm{sc}}}{r_{\text {sun } / \mathrm{sc}}^{3}}-\frac{\vec{r}_{\text {sun } / \text { earth }}}{r_{\text {sun/earth }}^{3}}\right), \\
\vec{a}_{\text {moon }} & =\mu_{\text {moon }}\left(\frac{\vec{r}_{\text {moon/sc }}}{r_{\text {moon } / \mathrm{sc}}^{3}}-\frac{\vec{r}_{\text {moon/earth }}}{r_{\text {moon/earth }}^{3}}\right), \\
\vec{a}_{\mathrm{srp}} & =C_{\text {srp }} \frac{S\left(1+c_{\mathrm{refl}}\right)}{2 m} \frac{\vec{r}_{\mathrm{sc} / \mathrm{sun}}}{r_{\mathrm{sc} / \mathrm{sun}}} \\
\vec{a}_{J_{2}} & =\frac{3 \mu J_{2} \rho_{\mathrm{E}}^{2}}{2 r^{5}}\left(\left(5 \frac{\left(\vec{r} \cdot \hat{k}_{\mathrm{E}}\right)}{r^{2}}-1\right) \vec{r}-2\left(\vec{r} \cdot \hat{k}_{\mathrm{E}}\right) \hat{k}_{\mathrm{E}}\right),
\end{aligned}
$$

where $\overrightarrow{(\cdot)}$ denotes a coordinate-free (unresolved) vector, $\mu_{\text {sun }}$ and $\mu_{\text {moon }}$ are the gravitational constants of the sun and moon, $C_{\mathrm{srp}}$ is the solar radiation pressure constant, $S$ is the solar-facing surface area, $c_{\text {reff }}$ is the surface reflectance, $\rho_{\mathrm{E}}$ is Earth's equatorial radius, $\hat{k}_{\mathrm{E}}$ is the $z$-axis unit vector of the Earth-centered inertial frame, and $J_{2}$ is the dominant coefficient in the considered geopotential perturbation model, where additional higher order terms are ignored. The sum of the individual disturbance accelerations yields the total disturbance acceleration considered in (1). Figure 1 shows an annual time history of the disturbance force components for a $4000 \mathrm{~kg}$ satellite in GEO.
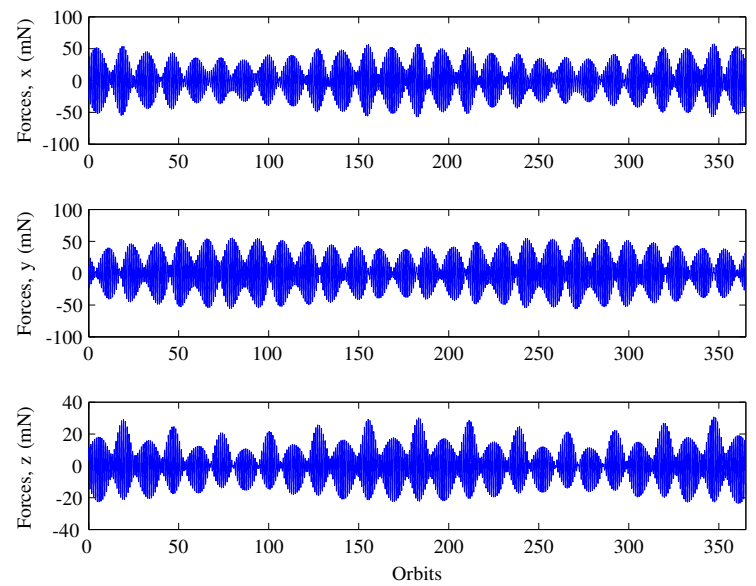

Fig. 1: Annual disturbance force components for a 4000kg satellite in geostationary orbit.

For MPC design, we linearize (1). For small maneuvers around a nominal circular orbit, the linearized $\mathrm{CWH}$ equations [15], [16] approximate the relative motion as

$$
\begin{aligned}
\delta \ddot{x}-3 n^{2} \delta x-2 n \dot{y} & =\frac{F_{x}}{m}+a_{\mathrm{p}, x}, \\
\delta \ddot{y}+2 n \delta \dot{x} & =\frac{F_{y}}{m}+a_{\mathrm{p}, y}, \\
\delta \ddot{z}+n^{2} \delta z & =\frac{F_{z}}{m}+a_{\mathrm{p}, z},
\end{aligned}
$$

where $\delta x, \delta y$ and $\delta z$ are the components of the position vector of the spacecraft relative to the nominal location, $F_{x}, F_{y}, F_{z}$ are the thrust force vector components, $a_{\mathrm{p}, x}, a_{\mathrm{p}, y}, a_{\mathrm{p}, z}$ are the perturbation acceleration vector components, and $n=\sqrt{\mu / R_{0}^{3}}$ is the motion of the nominal orbit.

We parameterize the attitude-error rotation matrix $\tilde{R} \triangleq$ $R^{\mathrm{T}} R_{\mathrm{d}}$ using the set of 3-2-1 Euler angles $(\psi, \theta, \phi)$ as $\tilde{R}=C_{1}(\phi) C_{2}(\theta) C_{3}(\psi)$, where $R_{\mathrm{d}}$ is the desired attitude trajectory, and $C_{1}, C_{2}$, and $C_{3}$ are elementary rotations about the $x, y$, and $z$-axes by $\psi, \theta$, and $\phi$, respectively. The linearization of the attitude dynamics and kinematics about an equilibrium $y$-axis (principal axis) spin with an angular rate corresponding to the mean motion $n$ of the orbit yields

$$
\begin{array}{rlrl}
J_{1} \dot{\delta} \omega_{1}=- & \left(J_{2}-J_{3}+\alpha_{2}-\alpha_{3}\right) n \delta \omega_{3}+n \alpha_{3} \delta \nu_{3} \\
& -\alpha_{1} \eta_{1}+\tau_{1}, & \\
J_{2} \dot{\delta} \omega_{2}=- & \alpha_{2} \eta_{2}+\tau_{2}, & \\
J_{3} \dot{\delta} \omega_{3}=- & \left(J_{1}-J_{2}+\alpha_{1}-\alpha_{2}\right) n \delta \omega_{1}-n \alpha_{1} \delta \nu_{1} \\
& \quad-\alpha_{3} \eta_{3}+\tau_{3}, & \\
\delta \dot{\nu}_{1}= & \eta_{1}, & \delta \dot{\phi}=\delta \omega_{1}+n \delta \psi, \\
\delta \dot{\nu}_{2}= & \eta_{2}, & \delta \dot{\theta}=\delta \omega_{2}, \\
\delta \dot{\nu}_{3}= & \eta_{3}, & & \delta \dot{\psi}=\delta \omega_{3}-n \delta \phi .
\end{array}
$$


Without orbital correction maneuvers, due to the disturbance accelerations (2), a spacecraft in GEO will drift from its assigned orbital position. In addition, the spacecraft uses its reaction wheels to compensate for any torque disturbances, thus maintaining a nadir-pointing configuration. Without any momentum management, the wheels' angular momentum may saturate, and the spacecraft may lose the capability to compensate for any disturbance torques.

The objective of the controller designed in this paper is to determine control inputs for the on-board thrusters and reaction wheels such that: $(R 1)$, the spacecraft maintains a nadir-pointing attitude while the reaction wheels are brought to rest, $(R 2)$, the spacecraft remains in a specified station keeping window, $(R 3)$, the fuel consumption is minimized, and $(R 4)$, the control produces forces and torques that can be simultaneously realized using the same set of thrusters.

\section{BASIC MPC FOR LOW THURST SK-MM}

Based on the linearized attitude and orbital dynamics model (3), (4) it is relatively easy to design a basic MPC for SK-MM, which extends an LQR design to account for the nadir-pointing requirement $(R I)$ and bounds on forces and torques due to maximum thrust magnitude.

\section{A. Design of basic MPC for SK-MM}

For MPC design, (3) and (4) are sampled with period $T_{s}$ to obtain the discrete-time linear prediction model

$$
x(t+1)=A x(t)+B u(t),
$$

where $x=\left[\begin{array}{lllllllllllllll}\delta x & \delta y & \delta z & \delta \dot{x} & \delta \dot{y} & \delta \dot{z} & \delta \phi & \delta \theta & \delta \psi & \delta \omega_{1} & \delta \omega_{2} & \delta \omega_{3}\end{array}\right.$ $\left.\begin{array}{llll}\delta \nu_{1} & \delta \nu_{2} & \delta \nu_{3}\end{array}\right]^{\mathrm{T}}, u=\left[\begin{array}{lllllllll}F_{x} & F_{y} & F_{z} & \eta_{1} & \eta_{2} & \eta_{3} & \tau_{1} & \tau_{2} & \tau_{3}\end{array}\right]^{\mathrm{T}}$, are the state and control input vectors, respectively. We do not include the disturbances (2) in (5) because of their highly nonlinear form.

Due to the nadir-pointing requirement $(R 1)$, we constrain the Euler angles to remain within the allowed tolerances

$$
|\delta \phi| \leq \delta \phi_{\max }, \quad|\delta \theta| \leq \delta \theta_{\max }, \quad|\delta \psi| \leq \delta \psi_{\max } .
$$

We also introduce bounds on forces, torques, and the angular accelerations of the reaction wheels, yielding

$$
u_{\min } \leq u \leq u_{\max }
$$

While constraint (7) accounts for the maximum input magnitudes, we assume that the thrusters can generate all requested forces and torques, i.e., we ignore the force-torque coupling induced by utilizing the same thrusters for SK-MM.

Using (5)-(7), the finite-horizon optimal control problem of MPC with a linear-quadratic cost function is given by

$$
\begin{array}{cl}
\min _{U_{t}} & x_{N \mid t}^{\mathrm{T}} P x_{N \mid t}+\sum_{k=0}^{N-1} x_{k \mid t}^{\mathrm{T}} Q x_{k \mid t}+u_{k \mid t}^{\mathrm{T}} R u_{k \mid t}, \\
\text { s.t. } & x_{k+1 \mid t}=A x_{k \mid t}+B u_{k \mid t} \\
& x_{0 \mid t}=x(t) \\
& u_{\min } \leq u_{k \mid t} \leq u_{\max } \\
& \delta \phi_{\min } \leq \delta \phi_{k \mid t} \leq \delta \phi_{\max } \\
& \delta \theta_{\min } \leq \delta \theta_{k \mid t} \leq \delta \theta_{\max } \\
& \delta \psi_{\min } \leq \delta \psi_{k \mid t} \leq \delta \psi_{\max }
\end{array}
$$

where $N$ is the prediction horizon, $U_{t}=\left[u_{0 \mid t}, \ldots, u_{N-1 \mid t}\right]$, $Q \geq 0, R>0$ are the stage cost weight matrices, and $P>0$ is the terminal cost weight [17], which is the solution of the discrete algebraic Riccati equation for (5), with $Q, R$ as in (8). At every sampling instant $t$, MPC computes the optimal solution $U_{t}^{*}$ of (8) for the current state $x(t)$ and applies $u(t)=u_{0 \mid t}^{*}$ to the spacecraft.

While $(R 1)$ is enforced via constraints (6), the MPC based on (8) enforces $(R 2)$ and $(R 3)$, which are the regulation performance and the control effort, by tuning the cost function weights, $Q, R$, respectively, similar to a classical LQR design. Finally, $(R 4)$ is assumed to be enforced a posteriori, e.g., by control allocation [18].

\section{B. Simulation results of basic MPC for $S K-M M$}

The results for an annual simulation of the spacecraft dynamics under the control of the MPC based on (8) are reported in Figure 2. Here, $T_{\max }=100 \mathrm{mN}$ is the maximum thrust for each thruster, used to compute the bounds in (7). Figure 2a shows that MPC achieves very accurate regulation performance, but this comes at the cost of very high fuel consumption $(\Delta v)$. In fact, [19] reports that for a positional accuracy of 0.05-0.1 degrees, out-of-orbital-plane SK requires between 41 and $51 \mathrm{~m} / \mathrm{s} /$ year, and in-orbital-plane SK requires $1.9 \mathrm{~m} / \mathrm{s} / \mathrm{year}$, while from Figure $2 \mathrm{~b}$, the MPC based on (8) requires $\Delta v_{z}=66.3 \mathrm{~m} / \mathrm{s} /$ year out-of-orbitalplane ( $z$-direction), while for in-orbital-plane, $\Delta v_{y}=174.7$ $\mathrm{m} / \mathrm{s} /$ year and $\Delta v_{x}=162.9 \mathrm{~m} / \mathrm{s} / \mathrm{year}$, (although the accuracy is almost three orders of magnitude higher).

As indicated in Section III-A, $(R 3)$ is achieved by tuning the cost function. However, an attempt to reduce fuel consumption by simply reducing $Q$ (or increasing $R$ ) reduces the regulation performance, leading to violation of $(R 2)$, and still does not achieve the desired range for annual $\Delta v$. We have found two reasons for this: $(i)$, the prediction model (5) neglects the disturbances (2), and (ii) the control effort of the MPC based on (8) is mostly linear with respect to the state. Due to $(i)$, the controller does not take advantage of the periodicity of the disturbances, and overreacts or under-reacts depending of the cost function weights. Also, MPC's nonlinear behavior is due to the constraints, and hence (ii) follows from the fact that in (8), the orbital dynamics are essentially unconstrained. While these results seem disappointing, they are a result of not exploiting the full potential of MPC. Next we show a slightly more advanced design that is aimed at resolving $(i)$ and $(i i)$.

\section{Advanced MPC FOR Low Thrust SK-MM}

The limitations of the design in Section III can be removed by exploiting some of the key features of MPC, namely the prediction of the dynamics and the capability of enforcing states and (combined) input constraints.

\section{A. Design of Advanced MPC for $S K-M M$}

To improve the prediction of MPC, we augment (5) with a prediction model of the disturbance accelerations (2),

$$
x(t+1)=A x(t)+B u(t)+\mathcal{O}_{\mathrm{H} / \mathrm{E}} a_{\mathrm{p}, t},
$$




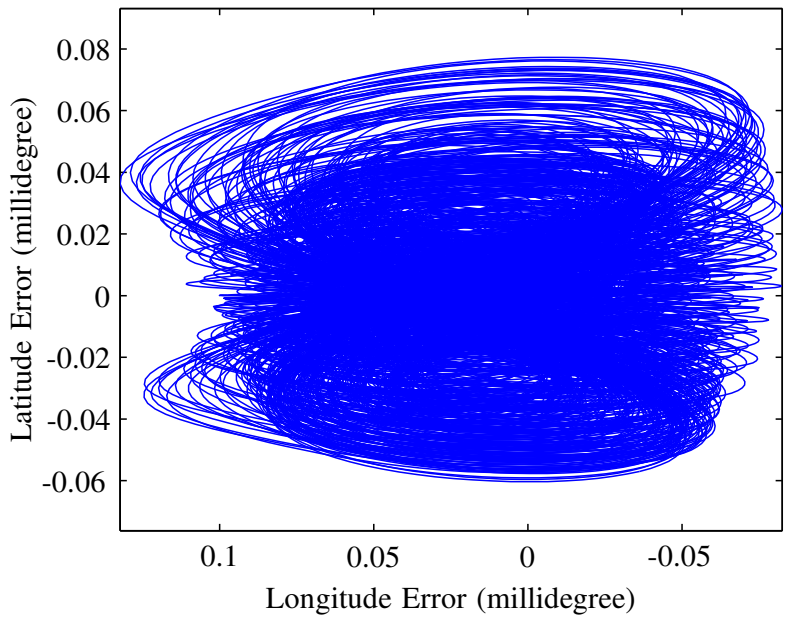

(a) Annual latitude and longitude.

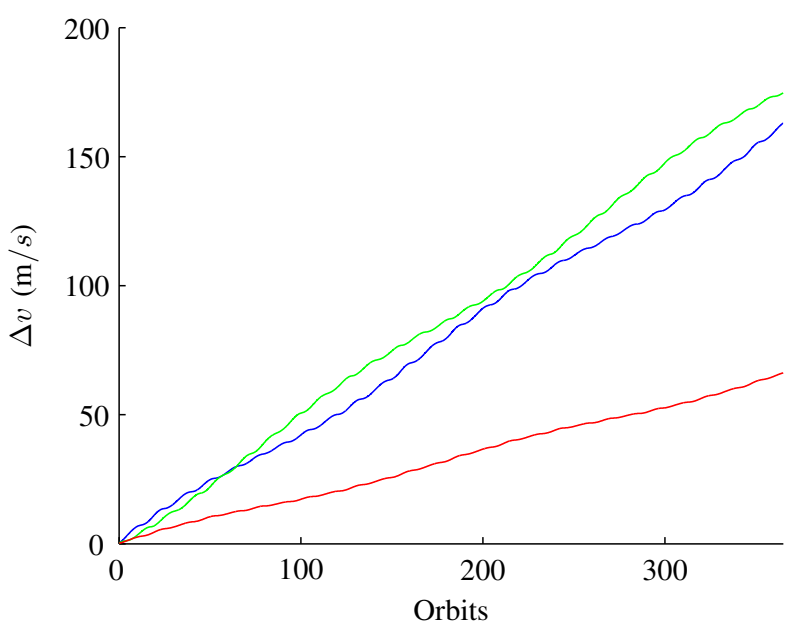

(b) Annual velocity increment.

Fig. 2: Annual simulation of spacecraft in closed-loop with basic MPC for SK-MM.

where $a_{\mathrm{p}, t+k}$ is the total disturbance acceleration at time step $t$ at the desired nominal orbit, and $\mathcal{O}_{\mathrm{H} / \mathrm{E}}$ is the rotation matrix that transforms the components of $a_{\mathrm{p}, k}$ from the inertial frame into the components of the same acceleration in Hill's frame. In (9) we have approximated the disturbance assuming the spacecraft is at its nominal orbit. Thus, the disturbance is obtained by integrating (2) along the nominal orbit, i.e., before solving the finite-time optimal control problem.

The error introduced in (9) by approximating the disturbance is relatively small if the spacecraft is close to its nominal orbit, which is guaranteed with proper station keeping. To further guarantee this, and to solve the second limitation of the MPC in Section III, we introduce SK window constraints that impose a limit on the error of the spacecraft orbit in terms of latitude and longitude,

$$
|\delta y| \leq r_{0} \tan \left(\lambda_{1, \max }\right), \quad|\delta z| \leq r_{0} \tan \left(\lambda_{2, \max }\right),
$$

where $\lambda_{1, \max }$ is the maximum tolerable longitude error, and $\lambda_{2, \max }$ is the maximum tolerable latitude error. As it will be discussed later, constraint (10) forces nonlinear behavior on SK control action, which can be leveraged to reduce the fuel consumption while keeping the spacecraft in the desired SK window.

Finally, by replacing (7) with slightly more complex constraints, we can also enforce (R4) and hence command torques and forces that can be generated by the single set of (low-thrust) thrusters, with thrust vector $T$ such that $\|T\|_{\infty} \leq T_{\max }$. For a spacecraft equipped with six dual-axis thrusters, assuming that the attitude error is small and that the spacecraft is in nadir-pointing configuration, (guaranteed by (6)), we introduce the constraints

$$
\left\|\left[\begin{array}{cc}
\Gamma & \Gamma \\
L & -L
\end{array}\right]^{-1}\left[\begin{array}{cc}
\mathcal{O}_{\mathrm{L} / \mathrm{H}} & 0 \\
0 & I
\end{array}\right]\left[\begin{array}{l}
F \\
\tau
\end{array}\right]\right\|_{\infty} \leq T_{\max },
$$

where $\Gamma, L, \mathcal{O}_{\mathrm{L} / \mathrm{H}}$ are appropriate matrices related to the geometry of the thruster locations in the spacecraft frame. The torques and forces that satisfy (11) can be achieved with the available thrust magnitude and thruster configuration.

Using (6), (9), (10), and (11) we obtain a more advanced MPC design, replacing (8) with

$$
\begin{array}{ll}
\min _{U_{t}} & x_{N \mid t}^{\mathrm{T}} P x_{N \mid t}+\sum_{k=0}^{N-1} x_{k \mid t}^{\mathrm{T}} Q x_{k \mid t}+u_{k \mid t}^{\mathrm{T}} R u_{k \mid t}, \\
\text { s.t. } \quad & x_{k+1 \mid t}=A x_{k \mid t}+B u_{k \mid t}+\mathcal{O}_{\mathrm{H} / \mathrm{E}} a_{\mathrm{p}, k \mid t} \\
& x_{0 \mid t}=x(t) \\
- & T_{\max } \leq D u_{k \mid t} \leq T_{\max } \\
& \delta y_{\min } \leq \delta y_{k \mid t} \leq \delta y_{\max } \\
& \delta z_{\min } \leq \delta z_{k \mid t} \leq \delta z_{\max } \\
& \delta \phi_{\min } \leq \delta \phi_{k \mid t} \leq \delta \phi_{\max } \\
& \delta \theta_{\min } \leq \delta \theta_{k \mid t} \leq \delta \theta_{\max } \\
& \delta \psi_{\min } \leq \delta \psi_{k \mid t} \leq \delta \psi_{\max }
\end{array}
$$

where the matrix $D$ is derived from (11). Due to the introduction of (10), the cost function weights in (12) can be selected to significantly penalize the control effort, thus avoiding any actuation until the predicted trajectory evolves outside the allowed SK window. In order to take advantage of the periodicity of (2), the time length of the MPC prediction horizon should be at least half an orbit. For SK-MM, the relatively fast attitude dynamics imposes constraints on the maximum sampling period. Hence, increasing the time length of the prediction horizon can be only achieved by increasing $N$, and hence the computational cost of solving (12).

\section{B. Simulation results of advanced MPC for SK-MM}

The results for an annual simulation of the spacecraft dynamics under the control of the MPC based on (12) are reported in Figure 3. Figure 3a shows that the spacecraft position error always resides in the allowed SK window, that is, $(R 2)$ is satisfied. Note that the trajectory fills the window, i.e., the controller uses almost the entire range of allowed SK error. As opposed to the design in Section III, Figure $3 \mathrm{~b}$ shows that the advanced MPC design based on (12) requires much less $\Delta v$. In detail, in the out-of-plane $z$-direction, $\Delta v_{z}=59 \mathrm{~m} / \mathrm{s} /$ year, while in the orbital plane, $\Delta v_{y}=1.6$ $\mathrm{m} / \mathrm{s} /$ year and $\Delta v_{x}=0.45 \mathrm{~m} / \mathrm{s} /$ year. These values are very 
close to those presented in [19] (see Section III-B), where however, the SK window is 5-10 times larger than the one considered here.

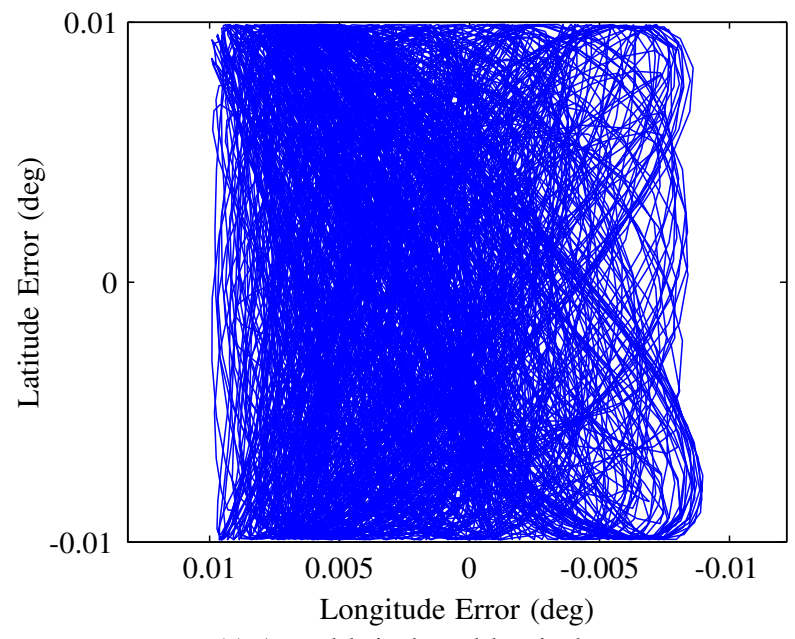

(a) Annual latitude and longitude
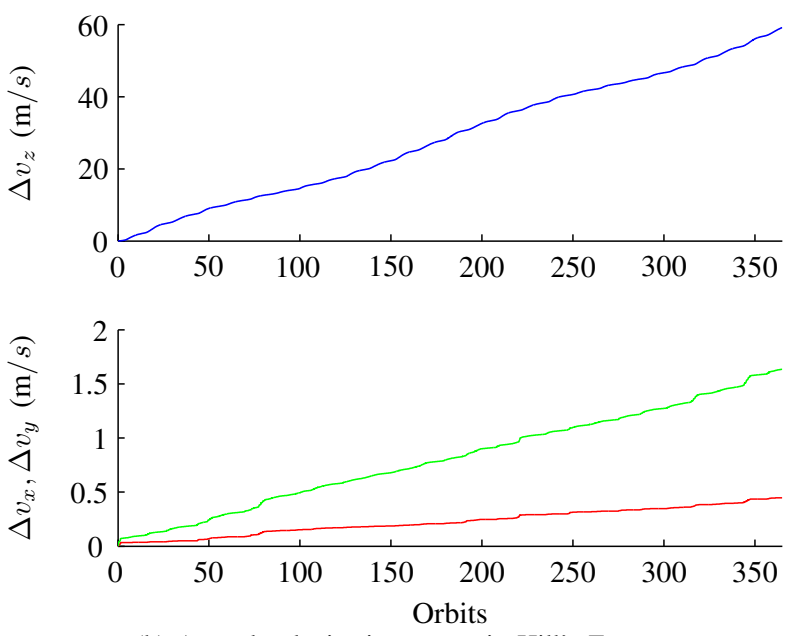

(b) Annual velocity increment in Hill's Frame.

Fig. 3: Annual simulation of spacecraft in closed-loop with advanced MPC for SK-MM.

The coupling between forces and torques is demonstrated by the results reported in Figure 4, where the MPC based on (12), although with different cost function weights from the ones used in the annual simulation, aggressively regulates the SK error and reduces the speed of the reaction wheels. Figure 4 shows that the controller first commands torques to dump the momentum of the reaction wheels. The spacecraft thus avoids any gyroscopic effects due to momentum stored in axes not coincident with the orbital rotation axis, which would lead to Euler angle constraint violations. Then, the controller commands forces so that the SK error is regulated to zero. Such complementary behavior is a result of constraint (11), which assures the feasibility of simultaneously realizing the requesting forces and torques using a single set of thrusters.

\section{Discussion}

The MPC design presented in Section IV is not significantly more complex than the one introduced in Section III. The novel elements are the disturbance prediction, which is a constant vector that can be pre-computed, and the SK window and the torque-force constraints, which are additional state and input constraints, respectively. Thus, the change in terms of performance may be surprising.

However, if we look at the modifications, they address the two main sources of excessive fuel consumption for the MPC in Section III. By introducing constraints on the SK window, and increasing the cost function weight on the control effort, the MPC policy avoids large actuation until the spacecraft is predicted to leave the SK window. This achieves $(R 2)$ without excessive penalty on $(R 3)$. When this is coupled with the disturbance prediction, which allows the controller to exploit situations when the disturbance "eventually works in the controller favor," the gains in terms of fuel consumptions are fully justified. The force-torque constraint guarantees that the desired forces and torques are actually achievable with the available propulsion system, so that control allocation becomes trivial.

It is indeed surprising, however, that the MPC based on (12) achieves fuel consumption very close to that reported in [19], especially as it has a SK window 5-10 times smaller, since MPC uses a limited knowledge of the future, applies a continuous feedback, and, in terms of computational cost, results in a quadratic program of limited complexity, to the point that it can be solved quickly even by low complexity and easy-to-verify iterative algorithms [11]. In fact, this is the real potential of MPC in low thrust SK-MM: being able to extract a complex high performance solution despite a relatively simple design and with limited computational load.

\section{CONCLUSIONS}

Model predictive control has shown significant potential for spacecraft control applications, due to its capability of handling multivariable constrained systems. By exploiting enforcement of constraints and predictive capabilities, together with an appropriate design of the cost function, MPC can achieve high performance in spacecraft control applications with a computational load that is feasible for on-board implementation. Thus, MPC is a valid candidate to meet tight mission requirements and increase spacecraft autonomy.

We have demonstrated such potential in a case study of simultaneous SK-MM for all-electric spacecraft. The MPC design achieves fuel consumption similar to that of open-loop optimal control techniques, while achieving 5-10 times more precise spacecraft positioning. Additionally, the MPC policy can coordinate the same set of thrusters to, simultaneously, generate forces for SK and torques for MM. The proposed MPC approach with some modifications can be also applied in more complex situations, such as in non-circular Halo orbits around the Earth-Moon L2 Lagrange point [20]. 

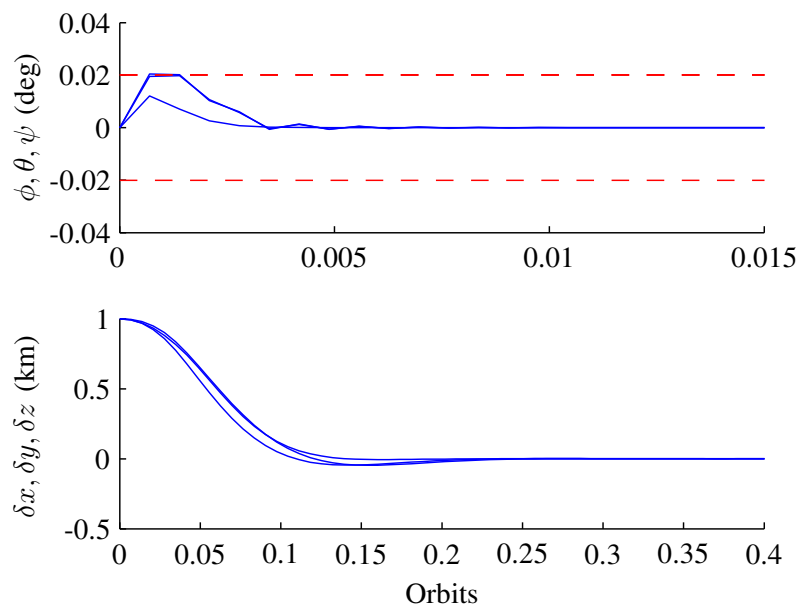

(a) Time histories of Euler angles and relative positions.
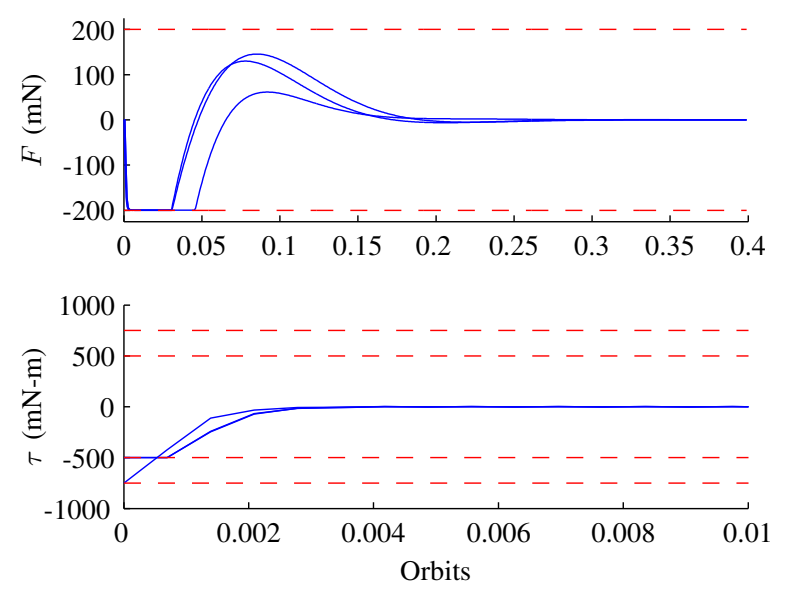

(b) Time histories of spacecraft forces and torques.
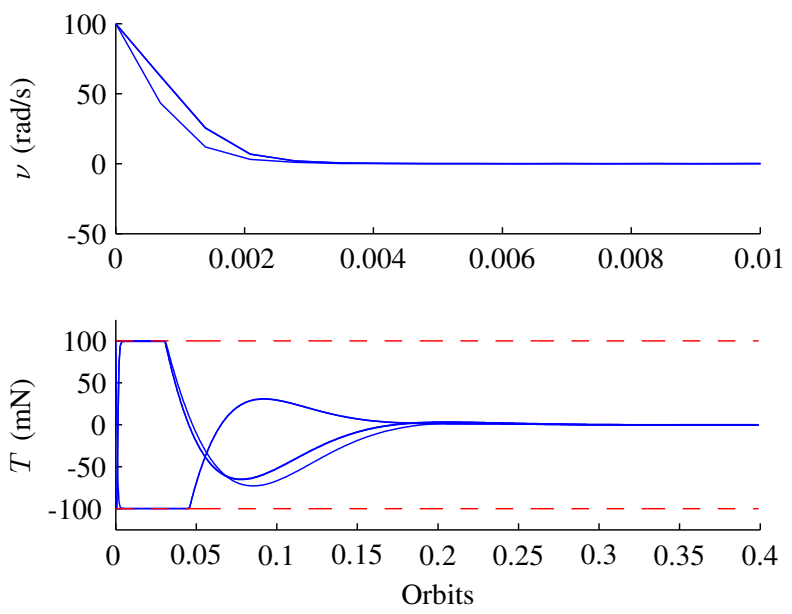

(c) Time histories of spacecraft reaction-wheel angular velocities and thruster magnitudes.

Fig. 4: Simulation of aggressive SK-MM maneuver for spacecraft in closed-loop with advanced MPC.

\section{REFERENCES}

[1] T. N. Edelbaum, "Optimum low-thrust rendezvous and station keeping," AIAA Journal, vol. 2, no. 7, pp. 1196-1201, 1964.

[2] E. M. Soop, Handbook of Geostationary Orbits. Springer, 1994.

[3] D. Hrovat, S. Di Cairano, H. E. Tseng, and I. V. Kolmanovsky, "The development of model predictive control in automotive industry: A survey," in IEEE Conf. Control Applications. IEEE, 2012, pp. 295302 .

[4] S. Di Cairano, "An industry perspective on MPC in large volumes applications: Potential benefits and open challenges," in Proc. 4th IFAC Nonlinear Model Predictive Control Conference. Citeseer, 2012, pp. $52-59$.

[5] E. Hartley, "Model predictive control for spacecraft rendezvous," Ph.D. dissertation, University of Cambridge, UK, 2010.

[6] S. Di Cairano, H. Park, and I. Kolmanovsky, "Model predictive control approach for guidance of spacecraft rendezvous and proximity maneuvering," Int. J. Robust and Nonlinear Control, vol. 22, no. 12, pp. 1398-1427, 2012.

[7] A. Weiss, I. Kolmanovsky, M. Baldwin, and R. S. Erwin, "Model predictive control of three dimensional spacecraft relative motion," in Proceedings of the American Control Conference, Montreal, Canada, June 2012, pp. 173- 178.

[8] Ø. Hegrenæs, J. T. Gravdahl, and P. Tøndel, "Spacecraft attitude control using explicit model predictive control," Automatica, vol. 41, no. 12, pp. 2107-2114, 2005.

[9] L. Breger, G. Inalhan, M. Tillerson, and J. P. How, "Cooperative spacecraft formation flying: Model predictive control with open-and closed-loop robustness," Elsevier Astrodynamics Series, vol. 1, pp. 237-277, 2007.

[10] J. A. Starek and I. V. Kolmanovsky, "Nonlinear model predictive control strategy for low thrust spacecraft missions," Optimal Control Applications and Methods, vol. 35, no. 1, pp. 1-20, 2014.

[11] S. Di Cairano, M. Brand, and S. A. Bortoff, "Projection-free parallel quadratic programming for linear model predictive control," International Journal of Control, vol. 86, no. 8, pp. 1367-1385, 2013.

[12] G. R. Cross, M. A. Potter, J. D. Whitehead, and J. T. Smith, "TDRSS momentum unload planning," Telematics and Informatics, vol. 8, no. 4, pp. 253-265, 1991.

[13] D. Losa, "High vs low thrust station keeping maneuver planning for geostationary satellites," Ph.D. dissertation, École Nationale Supérieure des Mines de Paris, 2007.

[14] A. Weiss, I. Kolmanovsky, D. S. Bernstein, and A. Sanyal, "Inertiafree spacecraft attitude control using reaction wheels," Journal of Guidance, Control, and Dynamics, vol. 36, no. 5, pp. 1425-1439, 2013.

[15] W. Clohessy and R. Wiltshire, "Terminal guidance system for satellite rendezvous," J. Aerospace Sci, vol. 27, no. 9, pp. 653-658, 1960.

[16] B. Wie, Space Vehicle Dynamics and Control, 2nd ed. Reston, VA: AIAA, 2008.

[17] S. Di Cairano and A. Bemporad, "Model predictive control tuning by controller matching," IEEE Tr. Automatic Control, vol. 55, no. 1, pp. 185-190, 2010.

[18] W. C. Durham, "Constrained control allocation," Journal of Guidance, Control, and Dynamics, vol. 16, no. 4, pp. 717-725, 1993.

[19] M. Martinez-Sanchez and J. E. Pollard, "Spacecraft electric propulsion-an overview," Journal of Propulsion and Power, vol. 14, no. 5 , pp. 688-699, 1998.

[20] U. Kalabic, A. Weiss, S. Di Cairano, and I. Kolmanovsky, "Stationkeeping and momentum-management on halo orbits around L2: Linear-quadratic feedback and model predictive control approaches," in Proc. AAS Space Flight Mechanics Meeting, Williamsburg, VA, AAS 15-307, 2015. 